\title{
Vision-based Target Localization from a Fixed-wing Miniature Air Vehicle
}

Joshua D. Redding

Scientific Systems Company, Inc., joshua@ssci.com

Timothy W. McLain

Brigham Young University - Provo, mclain@byu.edu

Randal W. Beard

Brigham Young University - Provo, beard@byu.edu

Clark N. Taylor

Brigham Young University - Provo

Follow this and additional works at: https://scholarsarchive.byu.edu/facpub

Part of the Electrical and Computer Engineering Commons, and the Mechanical Engineering

Commons

\section{Original Publication Citation}

Redding, J., McLain, T., Beard, R., and Taylor, C. Vision-based Target Localization from a Fixedwing Unmanned Aerial Vehicle, Proceedings of the American Control Conference, pp.

2862-2867, June 2006, Minneapolis, Minnesota.

\section{BYU ScholarsArchive Citation}

Redding, Joshua D.; McLain, Timothy W.; Beard, Randal W.; and Taylor, Clark N., "Vision-based Target Localization from a Fixed-wing Miniature Air Vehicle" (2006). Faculty Publications. 1537.

https://scholarsarchive.byu.edu/facpub/1537

This Peer-Reviewed Article is brought to you for free and open access by BYU ScholarsArchive. It has been accepted for inclusion in Faculty Publications by an authorized administrator of BYU ScholarsArchive. For more information, please contact ellen_amatangelo@byu.edu. 


\title{
Vision-based Target Localization from a Fixed-wing Miniature Air Vehicle
}

\author{
Joshua D. Redding Timothy W. McLain Randal W. Beard Clark N. Taylor
}

\begin{abstract}
This paper presents a method for localizing a ground-based object when imaged from a small fixed-wing unmanned aerial vehicle (UAV). Using the pixel location of the target in an image, with measurements of UAV position and attitude, and camera pose angles, the target is localized in world coordinates. This paper presents a study of possible error sources and localization sensitivities to each source. The localization method has been implemented and experimental results are presented demonstrating the localization of a target to within $11 \mathrm{~m}$ of its known location.
\end{abstract}

\section{INTRODUCTION}

Unmanned vehicles are prime candidates for tasks involving risk and repetition, or what the military calls the "dull, dirty and dangerous" [1]. The simplified goal of many of these tasks is to image and/or locate a target for tracking, reconnaissance, or delivery purposes. Therefore the ability to accurately determine the location of a ground-based object using aerial images would contribute to the success of these tasks. This paper presents a method of determining the location of an object in world/inertial coordinates using a gimballed camera on-board a small, fixed-wing UAV. Many of the current approaches to this localization problem involve imaging a target from a stationary air vehicle, i.e. a blimp or rotorcraft [2], [3]. Due to low-altitude and low-velocity flight capabilities, these aircraft allow significant simplification of the problem. However, blimps are not well suited for use in high winds or inclement weather, and the costs and complexities associated with rotor craft are high. It is therefore reasonable to explore localization methods involving more robust and less-expensive UAV platforms, such as fixed-wing UAVs.

While they lack the ability to hover, fixed-wing UAVs present unique benefits such as adaptability to adverse weather, a shorter learning curve for the untrained operator and extreme durability in harsh environments. Also, minimum airspeed requirements associated with fixed-wing aircraft can provide images from multiple vantage points, allowing for more robust localization.

Vision-based localization is well understood. However, much of the current research involves unmanned ground vehicles and controlled laboratory settings [4], [5]. Other research involves high-fidelity simulations, such as the work presented by Rysdyk [6]. Here a fixed-wing UAV was simulated to maintain a constant line-of-sight with a ground-based target. Although emphasis was given to UAV path-planning,

J. Redding is with the Intelligent and Autonomous Systems Group, Scientific Systems Company, Inc., Woburn, MA 01801, USA joshualssci.com

T. McLain is with the Department of Mechanical Engineering, Brigham Young University, Provo, UT 84602, USA mclain@byu.edu

R. Beard and C. Taylor are with the Department of Electrical and Computer Engineering, Brigham Young University, Provo, UT 84602, USA beard@ee.byu.edu, taylor@ee.byu.edu
Rysdyk gives valuable insight into target localization from a fixed-wing UAV. Stolle [7] presents similar research with some useful details on camera control. Both Rysdyk and Stolle deal with pointing a UAV-mounted camera at a known target location.

The focus of this paper is on determining the location of targets using a UAV-mounted camera. We present a general approach for target localization, provide an analysis of possible error sources, and demonstrate the effectiveness of the approach with experimental results.

\section{TeChNiCAL APPROACH}

A simple projection camera model is shown in Figure 1. The point $q$ is the projection of the point $p_{\mathrm{obj}}^{c}$ onto the image plane in pixels $(i p)$, where $p_{\text {obj }}^{c}$ denotes the location of an object $p$ relative to the center of the camera. It is assumed that the location $q$ is known in pixels and we wish to calculate it in meters, so $p_{\mathrm{obj}}^{c}$ can be found using similar triangles. Trucco, et al. [8] show that the change from pixels to meters in the image frame is accomplished by

$$
\begin{aligned}
x_{i m} & =\left(-y_{i p}+0_{y}\right) S_{y} \\
y_{i m} & =\left(x_{i p}-0_{x}\right) S_{x},
\end{aligned}
$$

where $0_{x}$ and $0_{y}$ denote the $x$ and $y$ offsets to center the image origin from the upper-left hand corner. $S_{x}$ and $S_{y}$ denote the image scalars for image $y$ and $x$ directions respectively due to the change of axes from $i p$ to $i m$ coordinates.

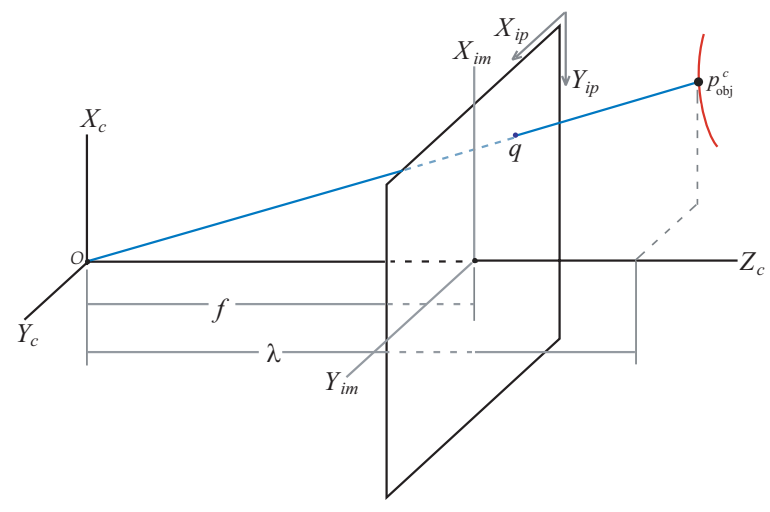

Fig. 1. Camera frames

If $q$ is converted into meters, it can be scaled into the camera frame using the law of similar triangles and the camera focal length, $f$. However, since the distance $\lambda$ from the camera center to $p_{\mathrm{obj}}^{c}$ is not known, scaling can only occur in two dimensions. For this reason, $\lambda$ is extracted and the 
scaling process is combined with (1) to form an expression for $p_{\mathrm{obj}}^{c}$ in terms of known pixel location $q$, as shown by

$$
p_{\text {obj }}^{c}=\lambda C^{-1} q,
$$

where $C$ is a matrix containing the scaling information for transformation between the camera, $c$, and the pixel, ip, coordinate frames. This is shown by

$$
\lambda q=\underbrace{\left[\begin{array}{ccc}
0 & f_{x} & 0_{x} \\
-f_{y} & 0 & 0_{y} \\
0 & 0 & 1
\end{array}\right]}_{C} p_{\mathrm{obj}}^{c}
$$

where $\frac{f}{S_{x}}=f_{x}$ and $\frac{f}{S_{y}}=f_{y}$. This shows that an object can be localized when $\lambda$ is known. We will address the problem of finding $\lambda$ in section II-B, but first we introduce a few necessary coordinate frames to place the camera in the sky, as seen in Figures 2 and 3.

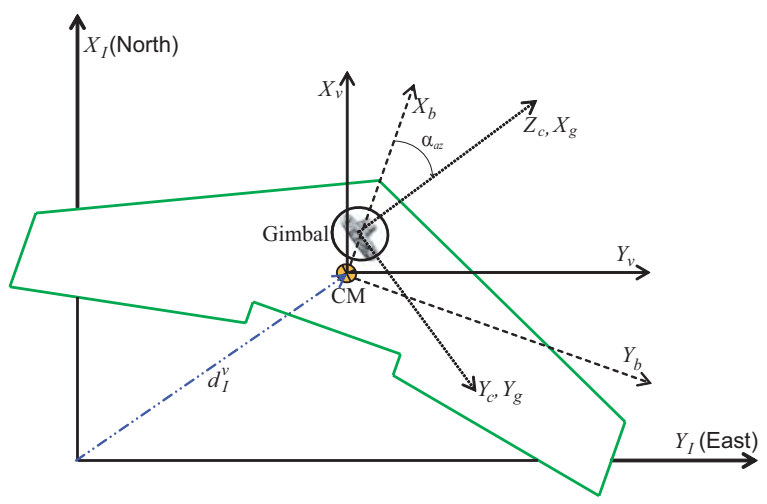

Fig. 2. Coordinate frames

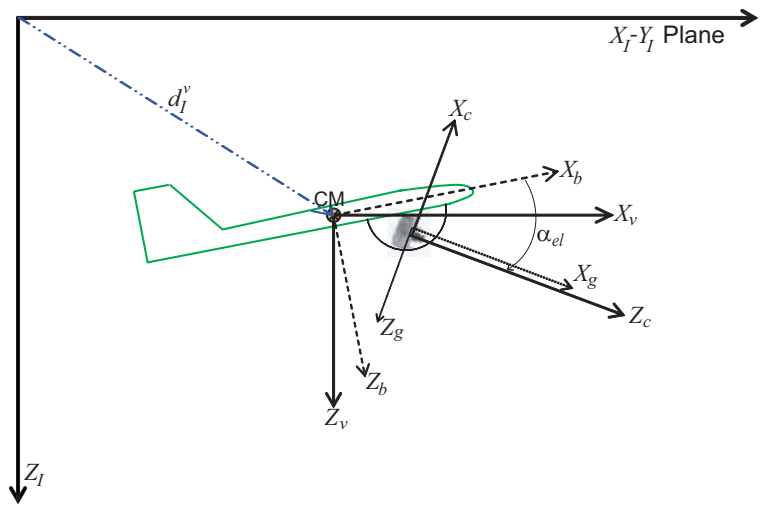

Fig. 3. Coordinate frames

All coordinate frames follow a right-hand rule. The camera frame $c$, has its origin at the camera's center, with the positive $\mathrm{Z}$-axis, $Z_{c}$, representing the optical axis of the camera. The origin of the gimbal frame $g$, is the center of the two-axis gimbal. The UAV body frame is centered at the UAV center of mass, with the X-axis, $X_{b}$, out the nose of the aircraft and the Y-axis, $Y_{b}$, out the right wing. The UAV vehicle frame $v$, is identical to the inertial frame $I$, only translated so the UAV center of gravity is its origin. As it will be necessary to move between coordinate frames frequently, a set of homogeneous transformation matrices are now introduced.

\section{A. Transformations}

A homogeneous transformation matrix (HTM) combines both rotation and translation between coordinate frames into a single matrix. The structure of an arbitrary HTM, $T_{i}^{j}$, is shown by

$$
T_{i}^{j}=\left[\begin{array}{cccc} 
& R & & -d_{i}^{j} \\
0 & 0 & 0 & 1
\end{array}\right],
$$

where $R$ represents a $(3 \times 3)$ rotation matrix and $d_{i}^{j}$ represents a $(3 \times 1)$ translation vector. Both rotation and translation occur from the $i^{\text {th }}$ to the $j^{\text {th }}$ coordinate frame. However, when written in this manner, the rotation occurs first, followed by the translation. Therefore the translation vector is resolved in the $j^{\text {th }}$ coordinate frame and negated to avoid requiring a pre-multiplication by $R_{i}^{j}$. The function of each HTM is described in Table I, and this section will discuss each individually, identifying elements and purpose.

TABLE I

HOMOGENEOUS TRANSFORMATION MATRICES.

\begin{tabular}{c|l}
\hline HTM & Description \\
\hline$T_{I}^{v}$ & Transformation from Inertial to UAV Vehicle frame \\
$T_{v}^{b}$ & Transformation from UAV Vehicle to UAV Body frame \\
$T_{b}^{g}$ & Transformation from UAV Body to Gimbal frame \\
$T_{g}^{c}$ & Transformation from Gimbal to Camera frame
\end{tabular}

1) Transformation $T_{I}^{v}$ : The transformation from the inertial to the vehicle frame is actually a single translation, therfore $T_{I}^{v}$ will only depend on the UAV's GPS location and barometric altitude measurements shown by

$$
\begin{aligned}
& T_{I}^{v}= {\left[\begin{array}{cc}
I & -d_{I}^{v} \\
0 & 1
\end{array}\right], \text { where } } \\
& d_{I}^{v}=\left[\begin{array}{c}
x_{\mathrm{UAV}} \\
y_{\mathrm{UAV}} \\
-h_{\mathrm{UAV}}
\end{array}\right],
\end{aligned}
$$

where $x_{\mathrm{UAV}}$ and $y_{\mathrm{UAV}}$ represent the North and East location of the UAV as measured by its GPS, and $h_{\mathrm{UAV}}$ represents the UAV's altitude as measured by a calibrated, on-board barometric pressure sensor.

2) Transformation $T_{v}^{b}$ : The transformation from the vehicle frame to the UAV body frame, $T_{v}^{b}$, consists of a single rotation based on measurements of Euler angles, shown by

$$
\begin{aligned}
T_{v}^{b} & =\left[\begin{array}{cc}
R_{v}^{b} & 0 \\
0 & 1
\end{array}\right], \text { where } \\
R_{v}^{b} & =\left[\begin{array}{ccc}
c_{\theta} c_{\psi} & c_{\theta} s_{\psi} & -s_{\theta} \\
s_{\phi} s_{\theta} c_{\psi}-c_{\phi} s_{\psi} & s_{\phi} s_{\theta} s_{\psi}+c_{\phi} c_{\psi} & s_{\phi} c_{\theta} \\
c_{\phi} s_{\theta} c_{\psi}+s_{\phi} s_{\psi} & c_{\phi} s_{\theta} s_{\psi}-s_{\phi} c_{\psi} & c_{\phi} c_{\theta}
\end{array}\right]
\end{aligned}
$$

and $\phi, \theta$ and $\psi$ represent the UAV's roll, pitch and heading angles in radians. Also, $c_{*}$ and $s_{*}$ abbreviate $\cos (*)$ and $\sin (*)$ respectively. 
3) Transformation $T_{b}^{g}$ : The transformation from the UAV body to the gimbal frame, $T_{b}^{g}$, will depend on the location of the UAV's center of mass with respect to the gimbal's rotation center. This vector, denoted by $d_{b}^{g}$, is resolved in the gimbal frame. $T_{b}^{g}$ will also depend on the rotation that aligns the gimbal's coordinate frame with the UAV's body frame. This rotation is denoted $R_{b}^{g}$ and requires measurements of the camera's azimuth and elevation angles $\alpha_{a z}$ and $\alpha_{e l}$ respectively, both of which are known. This transformation is shown by

$$
\begin{aligned}
T_{b}^{g} & =\left[\begin{array}{cc}
R_{b}^{g} & -d_{b}^{g} \\
0 & 1
\end{array}\right], \text { where } \\
R_{b}^{g} & =R_{y, \alpha_{e l}} R_{z, \alpha_{a z}} \\
= & {\left[\begin{array}{ccc}
c_{e l} & 0 & s_{e l} \\
0 & 1 & 0 \\
-s_{e l} & 0 & c_{e l}
\end{array}\right]\left[\begin{array}{ccc}
c_{a z} & s_{a z} & 0 \\
-s_{a z} & c_{a z} & 0 \\
0 & 0 & 1
\end{array}\right] } \\
= & {\left[\begin{array}{ccc}
c_{e l} c_{a z} & c_{e l} s_{a z} & s_{e l} \\
-s_{e l} & c_{a z} & 0 \\
-s_{e l} c_{a z} & -s_{e l} s_{a z} & c_{e l}
\end{array}\right], }
\end{aligned}
$$

where $d_{b}^{g}$ denotes the vector from the gimbal center to the UAV center of mass, $\alpha_{a z}$ denotes the azimuth angle of rotation about $Z_{g}$, and $\alpha_{e l}$ the elevation angle of rotation about $Y_{g}$, after $\alpha_{a z}$.

4) Transformation $T_{g}^{c}: T_{g}^{c}$ is the transformation from gimbal to camera reference frames. It will depend on the vector $d_{g}^{c}$, which describes the location of the gimbal's rotation center relative to the camera center and is resolved in the camera's coordinate frame. $T_{g}^{c}$ also depends on a simple rotation $R_{g}^{c}$, which aligns the camera's coordinate frame with that of the gimbal. It is shown by

$$
\begin{aligned}
T_{g}^{c}= & {\left[\begin{array}{cc}
R_{g}^{c} & -d_{g}^{c} \\
0 & 1
\end{array}\right], \text { where } } \\
R_{g}^{c}= & {\left[\begin{array}{ccc}
0 & 0 & -1 \\
0 & 1 & 0 \\
1 & 0 & 0
\end{array}\right], }
\end{aligned}
$$

since we chose $X_{c}=-Z_{g}$ and $Z_{c}=X_{g}$. Also, $d_{g}^{c}$ denotes the vector from the camera center to the gimbal center, resolved in camera frame.

We now have four HTMs that are based on a priori calibrations and real-time measurements from on-board sensors, which means we can freely move between coordinate frames as data is collected during flight. We now can extend (2) from the camera to the inertial frame, as shown by

$$
p_{\text {obj }}^{I}=\lambda\left[C T_{g}^{c} T_{b}^{g} T_{v}^{b} T_{I}^{v}\right]^{-1} q,
$$

where $p_{\text {obj }}^{I}$ denotes the object location in the world, or inertial frame. Knowing all other parameters on the right-hand side of (9), we are now ready to find the image depth, $\lambda$.

\section{B. Image Depth}

Image depth refers to the distance along the camera's optical axis, $Z_{c}$, to the object of interest in the image, and its value is usually unknown [9]. To estimate $\lambda$, the camera center is represented in inertial coordinates by $p_{\mathrm{cc}}^{I}$, as shown in Figure 4 and defined as

$$
p_{\mathrm{cc}}^{I}=\left[\begin{array}{c}
x \\
y \\
z \\
1
\end{array}\right]_{\mathrm{cc}}^{I}=\left[T_{g}^{c} T_{b}^{g} T_{v}^{b} T_{I}^{v}\right]^{-1}\left[\begin{array}{c}
x \\
y \\
z \\
1
\end{array}\right]_{\mathrm{cc}}^{c}
$$

where the vector $\left[\begin{array}{llll}x & y & z & 1\end{array}\right]_{\mathrm{cc}}^{c^{T}}$ is equal to $\left[\begin{array}{llll}0 & 0 & 0 & 1\end{array}\right]^{T}$, since it describes the location of the camera center in camera coordinates. Figure 4 also shows the location $q$, which was introduced in Figure 1. The location of $q$ in the inertial frame is described by the vector $\bar{p}_{\text {obj }}^{I}$, which is also depicted in Figure 4 and defined as

$$
\bar{p}_{\mathrm{obj}}^{I}=\left[\begin{array}{c}
\bar{x} \\
\bar{y} \\
\bar{z} \\
1
\end{array}\right]_{\mathrm{obj}}^{I}=\left[C T_{g}^{c} T_{b}^{g} T_{v}^{b} T_{I}^{v}\right]^{-1} q,
$$

where $q$ contains the target's pixel location, shown by

$$
q=\left[\begin{array}{llll}
x_{i p} & y_{i p} & 1 & 1
\end{array}\right]^{T} .
$$

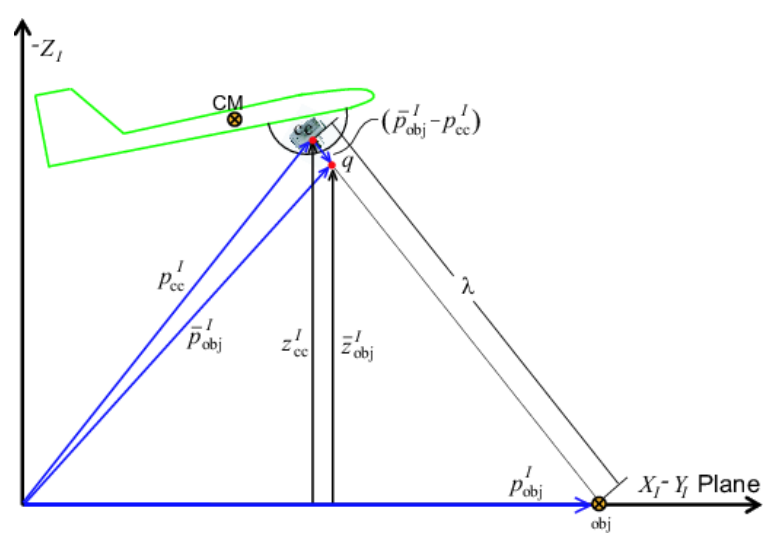

Fig. 4. Localization vectors

Referring again to Figure 4, and noting the implied assumption that the zero-altitude plane is defined where the UAV's altitude sensor was zeroed, the $z$ components of $\bar{p}_{\text {obj }}^{I}$ and $p_{\mathrm{cc}}^{I}$ form the relationship

$$
0=z_{\mathrm{cc}}^{I}+\lambda\left(\bar{z}_{\mathrm{obj}}^{I}-z_{\mathrm{cc}}^{I}\right) .
$$

The zero on the left-hand side of (13) follows from the assumption that the target lies on this plane of zero altitude. This assumption can be removed in the future as heightabove-ground, or terrain map measurements can provide information to make the left-hand side of (13) known, but non-zero. Since both $z_{\mathrm{cc}}^{I}$ and $\bar{z}_{\mathrm{obj}}^{I}$ are known from (10) and (11) respectively, (13) can easily be solved for $\lambda$ as

$$
\lambda=\frac{-z_{\mathrm{cc}}^{I}}{\left(\bar{z}_{\mathrm{obj}}^{I}-z_{\mathrm{cc}}^{I}\right)} .
$$

Since the inertial Z-axis, $Z_{I}$, is defined positive toward the center of the earth, $z_{\mathrm{cc}}^{I}$ will be negative for flight altitudes greater than the calibrated zero. Thus, (14) yields a positive value for $\lambda$, as expected. 


\section{Target Location}

Now that the depth of our current image is known, we can easily estimate the inertial location of the target in the image by

$$
p_{\mathrm{obj}}^{I}=\lambda\left[C T_{g}^{c} T_{b}^{g} T_{v}^{b} T_{I}^{v}\right]^{-1} q,
$$

or by continuing the method used to find $\lambda$, as shown by

$$
p_{\mathrm{obj}}^{I}=p_{\mathrm{cc}}^{I}+\lambda\left(\bar{p}_{\mathrm{obj}}^{I}-p_{\mathrm{cc}}^{I}\right) .
$$

We see that the localization of a visible target can be accomplished using only a camera and readily accessible UAV information however, noise and parameter uncertainty can significantly affect the quality of this localization estimate. Section III presents a study of error sources and their effects on target localization.

\section{ERROR ANALYSIS}

Like all aircraft, UAVs are susceptible to outside influences including wind gusts and variations in atmospheric pressures, air densities and temperatures. These phenomena, among others, can add unwanted noise to aircraft sensors. This noise, combined with inherent sensor inaccuracies, contaminates each measurement of position, altitude, airspeed and heading as well as roll, pitch and yaw rates. The purpose of this section is to explore the main error sources in UAV and gimbal control and to study how each affects the localization result.

\section{A. Error Sources}

In the equation

$$
p_{\text {obj }}^{I}=\lambda\left[C T_{g}^{c} T_{b}^{g} T_{v}^{b} T_{I}^{v}\right]^{-1} q,
$$

each term introduces inaccuracies to the end result. Since $\lambda$ is calculated from measurements of UAV altitude, its associated errors will be accounted for through altitude uncertainty. Errors in the camera calibration matrix, $C$, originate in the calibration routine itself and will be neglected. The transformation $T_{g}^{c}$ depends on the location of the UAV center of mass with respect to the camera center, which is known within millimeters and can also be ignored. Similarly, $T_{b}^{g}$ will depend on camera gimbal angles, which are controlled via commercially available hobby servos. Such servos have been tested accurate to less than half a degree and precise to less than one fifth of a degree. It is important to note, however, that such performance characteristics are only valid when the servo is given sufficient time to reach its desired angle, which is on the order of $5 \mathrm{~ms} / \mathrm{deg}$. Although this is believed to be sufficiently fast for typical changes in desired gimbal angles, these will not be ignored in this study $T_{b}^{g}$.

$T_{v}^{b}$ introduces further inaccuracies through errors in UAV attitude estimation. Euler angles $\phi, \theta$, and $\psi$ are estimated from gyro measurements of roll, pitch and yaw rates as well as accelerometer readings with reference to the gravity vector [10]. Unfortunately, gyros tend to drift, causing accumulating errors in $\psi$. Estimates of $\phi$ and $\theta$ are generated by subtracting the gravity vector from accelerometer measurements, a technique which works well under static conditions. However, this subtraction yields degraded results when the UAV is experiencing accelerations common during flight. Through laboratory tests, $\phi, \theta$ and $\psi$ have been shown to be statically accurate to within $5 \mathrm{deg}$, and dynamically accurate to within $10 \mathrm{deg}$.

The translation from inertial to vehicle frames, accomplished by $T_{I}^{v}$, adds inaccuracies that stem from both GPS measurements and barometric altitude readings. The major GPS inaccuracies are attributed to a variety of sources that combine to achieve an accuracy of roughly $10 \mathrm{~m}$ in the horizontal plane, and $25 \mathrm{~m}$ in the vertical plane [11]. For testing purposes, the known location of the target was measured using the GPS unit on the UAV. Since the bias portion of the GPS error equally effects measures of both UAV and target positions, it does not contribute to the error between the known location of the target and the estimated location of the target. Random errors in GPS measurements are average approximately $5 \mathrm{~m}$ in the horizontal plane. With the addition of an absolute pressure sensor, altitude inaccuracies are reduced to roughly $8 \mathrm{~m}$ [12].

TABLE II

UNCERTAINTIES, $U_{*}$

\begin{tabular}{c|c|c|c}
\hline Source & \pm Value & Source & \pm Value \\
\hline$\alpha_{a z}$ & $.5 \mathrm{deg}$ & $\alpha_{e l}$ & $.5 \mathrm{deg}$ \\
$\phi$ & $5 \mathrm{deg}$ & $\theta$ & $5 \mathrm{deg}$ \\
$\psi$ & $5 \mathrm{deg}$ & $x_{\mathrm{UAV}}$ & $5 \mathrm{~m}$ \\
$y_{\mathrm{UAV}}$ & $5 \mathrm{~m}$ & $h_{\mathrm{UAV}}$ & $8 \mathrm{~m}$ \\
$x_{i p}$ & 5 pixels & $y_{i p}$ & 5 pixels
\end{tabular}

The target pixel location, $q$, is also subject to uncertainties, including visual occlusions and lighting changes. Accounting for such, it is believed that $q$ can be trusted within about 5 pixels in both $x_{i p}$ and $y_{i p}$. Although actual uncertainties are not known, the values shown in Table II are the results of laboratory tests and it is assumed that they represent a $95 \%$ probability.

\section{B. Sensitivity and Propagation}

This section presents a study of localization sensitivity to uncertainties in measurements of UAV location and attitude as well as camera gimbal angles using the method of sequential perturbation [13]. The localization estimate of the target position can be expressed as a function of the UAV location and attitude and the gimbal azimuth and elevation angles according to

$$
\begin{aligned}
p_{\mathrm{obj}}^{I} & =\lambda\left[C T_{g}^{c} T_{b}^{g} T_{v}^{b} T_{I}^{v}\right]^{-1} q \\
& =F\left(\alpha_{a z}, \alpha_{e l}, \phi, \theta, \psi,(x, y, h)_{\mathrm{UAV}}\right) .
\end{aligned}
$$

Sequential perturbation is a numerical approach to estimate the propagation of uncertainties into a result and is used when direct calculation of partial derivatives is not feasible, as is the case with (18). Using sequential perturbation, sensitivities to errors in each variable are calculated under a nominal flight condition, in this case a large-orbit coordinated turn. These sensitivities are listed in Table III and show that errors in UAV roll angle and camera elevation angle most dramatically affect localization outcome. The fact that they are equally important is expected since during a localization flight the camera is panned to roughly $90 \mathrm{deg}$, which aligns $Y_{g}$, the axis about which camera elevation occurs, with $X_{b}$, the axis about which aircraft roll occurs. When aligned in this manner, the localization algorithm will not differentiate 

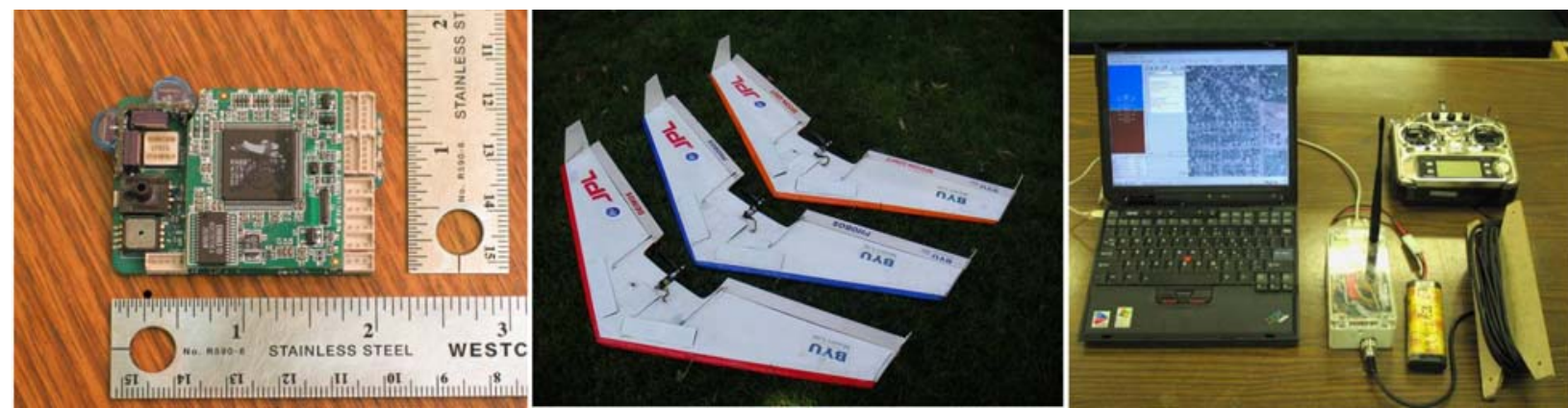

Fig. 5. (a) Kestrel autopilot. (b) Zagi airframes. (c) Ground station components.

between changes in elevation angle and changes in UAV roll angle.

TABLE III

NUMERICALLY APPROXIMATED PARTIAL DERIVATIVES

\begin{tabular}{c|c|c}
\hline$i$ & Parameter & $\partial F / \partial *$ \\
\hline 1 & $\alpha_{a z}$ & $1.1 \mathrm{~m} / \mathrm{deg}$ \\
2 & $\alpha_{e l}$ & $1.7 \mathrm{~m} / \mathrm{deg}$ \\
3 & $\phi$ & $1.7 \mathrm{~m} / \mathrm{deg}$ \\
4 & $\theta$ & $1.1 \mathrm{~m} / \mathrm{deg}$ \\
5 & $\psi$ & $0.8 \mathrm{~m} / \mathrm{deg}$ \\
6 & $x_{\text {UAV }}$ & $1.0 \mathrm{~m} / \mathrm{m}$ \\
7 & $y_{\text {UAV }}$ & $1.0 \mathrm{~m} / \mathrm{m}$ \\
8 & $h_{\text {UAV }}$ & $0.8 \mathrm{~m} / \mathrm{m}$ \\
9 & $x_{i p}$ & $0.15 \mathrm{~m} / \mathrm{pixel}$ \\
10 & $y_{i p}$ & $0.19 \mathrm{~m} / \mathrm{pixel}$
\end{tabular}

Using Table II in conjunction with Table III, the total expected localization error, $\Gamma$, can be computed [13] by

$$
\begin{aligned}
\Gamma & =\sqrt{\sum_{i=1}^{N}\left(\frac{\partial F}{\partial i} U_{i}\right)^{2}} \\
& =14.9 \mathrm{~m}
\end{aligned}
$$

where $i$ refers to each of the $N$ parameters on which $F$ is dependent, as listed in Table III. We can therefore conclude that it is theoretically possible to locate a target within 15 $\mathrm{m}$ using computer vision from a fixed-wing UAV under nominal flight conditions of $60 \mathrm{~m}$ altitude and a large-radius coordinated turn.

\section{Averaging Method}

Since each estimate of its location requires only one image of the target, it is theoretically possible to generate estimates at the frame rate of the camera. Although bandwidth constraints make this impossible, we can achieve several estimates per second, allowing for effective filtering to help reduce error. In this paper, we apply Recursive Least Squares (RLS) to filter the estimates.

Recursive Least Squares: Recursive Least Squares (RLS) is a simple method of recursively fitting a set of points to some function of choice by minimizing the sum of the squares of the offsets of the points. Typically, an RLS algorithm is used to fit a set of points to a characteristic line or quadratic, however, it can be also be used to find a characteristic point. In this case, the result of the RLS algorithm is identical to the result of a true average. The RLS algorithm implemented is detailed in Algorithm 1.

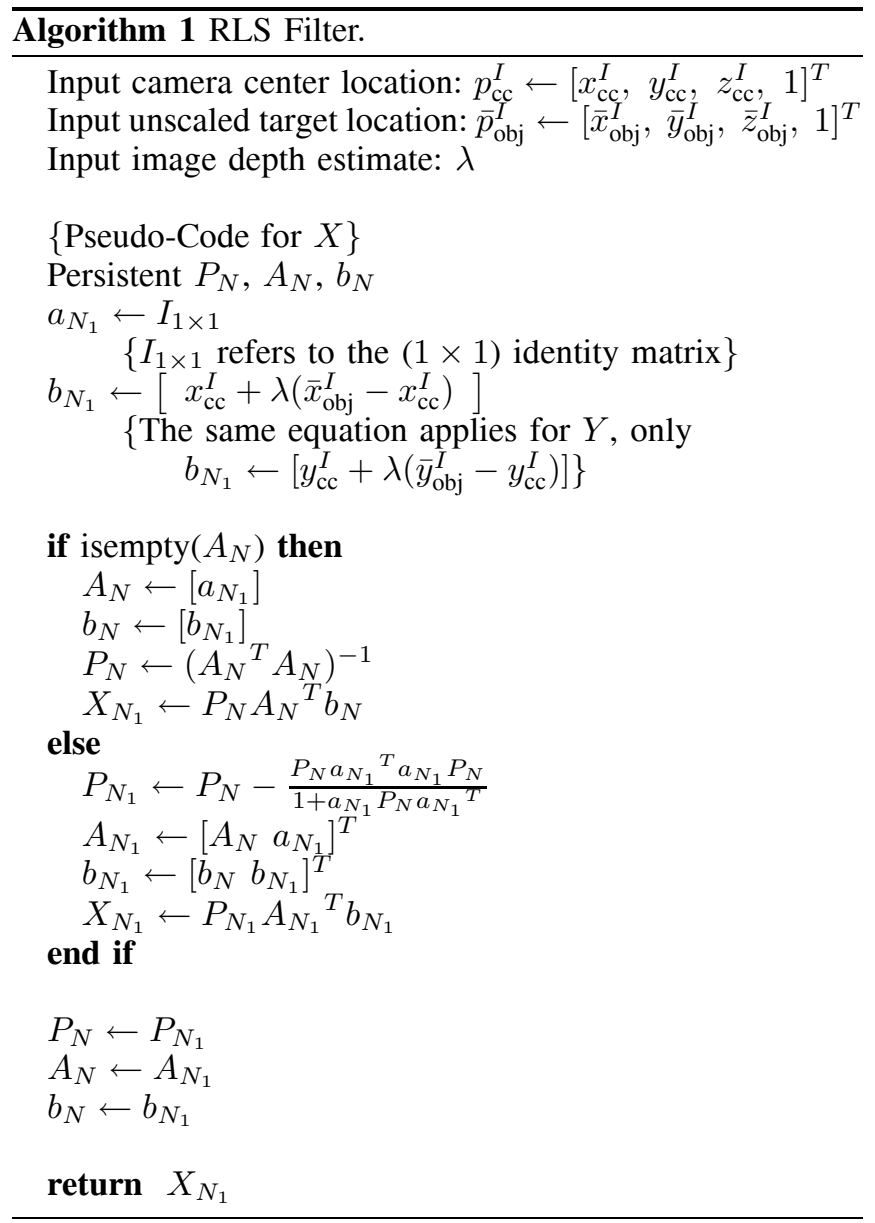

\section{Hardware TeStBed}

BYU has developed a reliable and robust platform for testing unmanned air vehicles [14]. Figure 5 shows the key elements of the testbed. The first frame shows BYU's Kestrel autopilot which is equipped with a Rabbit 3400 $29 \mathrm{MHz}$ processor, rate gyros, accelerometers, absolute and differential pressure sensors. The autopilot measures $3.8 \times 5.1 \times 1.9 \mathrm{~cm}$ and weighs 17 grams .

The second frame in Figure 5 shows the airframes used for the flight tests reported in this paper. The airframe is a 1.2 meter wingspan Zagi XS EPP foam flying wing, which was selected for its durability, ease of component installation, and flight characteristics. Embedded in the airframe are the 
Kestrel autopilot, batteries, a $1000 \mathrm{~mW}, 900 \mathrm{MHz}$ radio modem, a GPS receiver, a video transmitter, and a small analog camera.

The third frame in Figure 5 shows the ground station components. A laptop runs the Virtual Cockpit software that interfaces through a communication box to the UAVs. An $\mathrm{RC}$ transmitter is used as a stand-by fail-safe mechanism to facilitate safe operations.

\section{EXPERIMENTAL RESULTS}

The results of an initial hardware experiment are shown in Figure 6. The plot shows actual, estimated and filtered target locations from a UAV flying at $60 \mathrm{~m}$ altitude in a $50 \mathrm{~m}$ radius circular orbit around an initial guess of the target location. As can be seen in Figure 6, the filtered

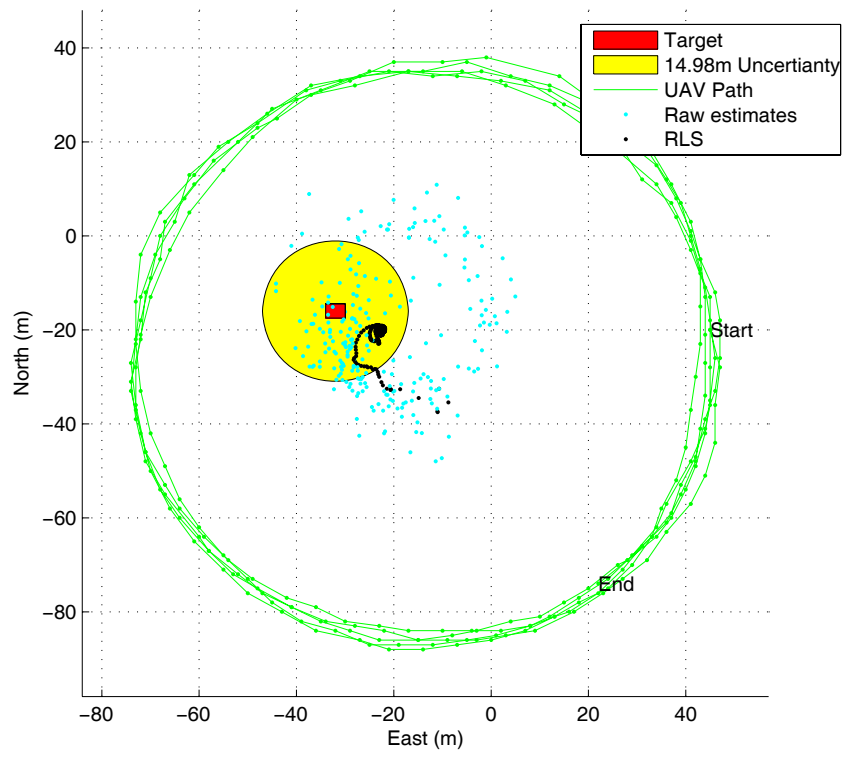

Fig. 6. Localization results

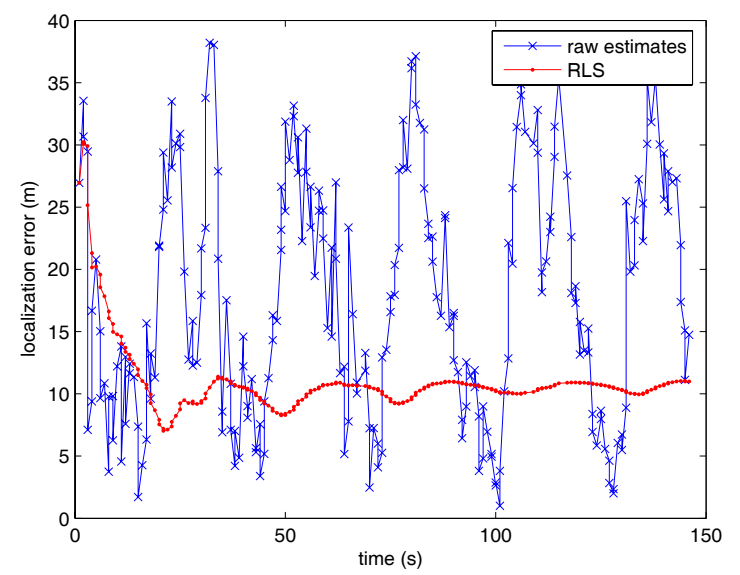

Fig. 7. Localization error

estimates of target location are nearly within the expected accuracy range of $15 \mathrm{~m}$. At the start, the first few estimates are outside the predicted error radius. However, they quickly move into it as more estimates are made and the target's position is known with more confidence. The time history of the localization error is shown in Figure 7 demonstrating the rapid convergence of the RLS estimate to a quasi steady-state error of $10.9 \mathrm{~m}$. In this case, RLS estimates converge within about $20 \mathrm{~s}$, which is the time required to fly about one half of an orbit. Two main factors are believed to contribute to the steady-state error: attitude estimation errors and lack of synchronization between attitude and position telemetry and vision data. Future efforts will attempt to reduce these error sources to further improve localization capabilities.

\section{CONCLUSIONS}

This paper demonstrates the feasibility of vision-based target localization from a small, fixed-wing UAV. Results from hardware implementation show that the method produces satisfactory results, with excellent prospects for future improvement. Localization estimates could be improved by increasing the accuracy of attitude estimates, most notably the UAV roll angle.

\section{ACKNOWLEDGMENTS}

This work was funded by AFOSR award number FA955004-1-0209 and the Utah State Centers of Excellence Program.

\section{REFERENCES}

[1] Office of the Secretary of Defense, Ed., Unmanned Aerial Vehicles Roadmap 2002-2027. Washington DC, USA: United States Government, 2002.

[2] L. Chaimowicz, B. Grocholsky, J. F. Keller, V. Kumar, and C. J. Taylor, "Experiments in Multirobot Air-Ground Coordination," in Proceedings of the 2004 International Conference on Robotics and Automation, New Orleans, LA, April 2004, pp. 4053-4058.

[3] R. Vidal and S. Sastry, "Vision-Based Detection of Autonomous Vehicles for Pursuit-Evasion Games," in IFAC World Congress on Automatic Control, Barcelona, Spain, July 2002.

[4] P. Saeedi, D. G. Lowe, and P. D. Lawrence, "3D Localization and Tracking in Unknown Environments," in Proceedings of the IEEE Conference on Robotics and Automation, vol. 1, Sept 2003, pp. 12971303.

[5] S. G. Chroust and M. Vincze, "Fusion of Vision and Inertial Data for Motion and Structure Estimation," Journal of Robotic Systems, vol. 21, pp. 73-83, January 2003.

[6] R. Rysdyk, "UAV Path Following for Constant Line-of-sight," in 2nd AIAA "Unmanned Unlimited" Systems, Technologies and Operations Aerospace, Land and Sea Conference, September 2003.

[7] S. Stolle and R. Rysdyk, "Flight Path Following Guidance for Unmanned Air Vehicles with Pan-Tilt Camera for Target Observation," in 22nd Digital Avionics Systems Conference, October 2003.

[8] E. Trucco and A. Verri, Introductory Techniques for 3-D Computer Vision. New Jersey, USA: Prentice-Hall, 2002.

[9] Y. Ma, S. Soatto, J. Kosecka, and S. S. Sastry, An Invitation to 3-D Vision From Images to Geometric Models. New York, USA: SpringerVerlag, 2003.

[10] D. B. Kingston and R. W. Beard, "Real-Time Attitude and Position Estimation for Small UAVs Using Low-Cost Sensors," in AIAA 3rd Unmanned Unlimited Systems Conference and Workshop, Chicago, Il, September 2004.

[11] Montana State University GPS Laboratory, “GPS Accuracy," 2004, http://www.montana.edu/ places/gps/lres357/slides/GPSaccuracy.ppt.

[12] Procerus Technologies, Inc., "Procerus UAV," 2005, http://www.procerusuav.com.

[13] R. S. Figliola and D. E. Beasley, Theory and Design for Mechanical Measurements. New York, USA: John Wiley and Sons, Inc., 2000.

[14] R. Beard, D. Kingston, M. Quigley, D. Snyder, R. Christiansen, W. Johnson, T. McLain, and M. Goodrich, "Autonomous vehicle technologies for small fixed wing UAVs," AIAA Journal of Aerospace Computing, Information, and Communication, vol. 2, no. 1, pp. 92108, January 2005. 